\title{
New Collaboration through Artefact-Mediated Interaction with a Joint Knowledge Base
}

\author{
Marco Bettoni ${ }^{1}$ and Eddie Obeng ${ }^{2}$ \\ ${ }^{1}$ Steinbeis Consulting Center Knowledge Management and Collaboration, Basel, Switzerland \\ 2Pentacle Virtual Business School, Beaconsfield, Buckinghamshire, United Kingdom \\ marco.bettoni@weknow.ch \\ Eddie Obeng@pentaclethevbs.com
}

\begin{abstract}
Collaboration is changing and increasingly emerging as what we call "New Collaboration", a knowledge-based and community-oriented way of working together (especially digital, online collaboration). Unfortunately, organisations use only a small percentage of the potential of New Collaboration. One main reason for this is that they do not understand that New Collaboration is based on knowledge sharing and requires the individual knowledge of the collaborators to be integrated into a shared knowledge structure, a so-called Joint Knowledge Base (JKB). This concept of a Joint Knowledge Base as the tacit knowledge structure which is constructed, shared and maintained during collaboration, emerged during the course of our previous work and became more and more prominent as a key to collaboration. When a group interacts, the JKB functions as an interaction bridge, and this is why it is a key to collaboration. In this paper, we will revise and elaborate in more detail our concept of a JKB and explain its role in artefact-mediated interaction. First, we will explain the main characteristics of New Collaboration and summarise them based on a concise definition. Secondly, we will introduce the concept of a Joint Knowledge Base, explore the role of social negotiation in constructing it, define the JKB as a distributed knowledge structure, discuss the problem of obstacles which hinder its development and suggest how to solve it by means of gaining deeper insight into the complexity of the involved processes (communication, interaction). And next we will further develop this solution by introducing the concept of boundary artefacts and describing their implementation as tools for artefact-mediated interaction by means of a systematic approach. Finally, we will explain this systematic approach and show how boundary artefacts and artefact-mediated interaction work in practice during meetings performed on a commercially available collaboration platform where they contribute to the construction of a JKB.
\end{abstract}

Keywords: new collaboration, knowledge sharing, joint knowledge base, boundary objects, artefact-mediated interaction

\section{Introduction}

The business world in which we are living today is a world of volatility, uncertainty, complexity and ambiguity (VUCA). It is a new world which changes faster than we can learn and where business outputs "depend on a wider and wider range of knowledge, skills, values, technologies and competences" (Obeng 1997, pp.3-9). In order to be successful in this new world, collaboration must adapt and change. In previous work, we proposed a knowledge-based and community-oriented understanding of collaboration which we called New Collaboration and described based on three models: a system model (Bettoni et al., 2016), a presence model (Bettoni et al., 2018) and a hierarchical process model (Bettoni and Obeng, 2020) called the "Pyramid of New Collaboration". This pyramid model of collaboration requires the individual knowledge of the collaborators to be integrated into a shared knowledge structure, a so-called "Joint Knowledge Base" (JKB). For doing this it is essential to understand how the JKB enables collaboration and specifically how the JKB is constructed and maintained. This is our main research question here. A better understanding of this aspect of knowledge sharing will contribute to raising awareness of the fact that a JKB plays an essential role in collaboration and to taking this seriously.

Secondly, it will help to understand how the interwoven process between knowledge sharing and collaboration works. Last but not least, it can help to understand group learning (and group work in general) not only as a form of knowledge acquisition (cognitive process) and participation in a social community (social process) but also as collaborative knowledge creation (Paavola et al., 2004). Therefore, the aim of this article is to revise and elaborate our concept of a JKB in more detail. Our results define the main characteristics of New Collaboration, specify the role of social negotiation in constructing the Joint Knowledge Base, define it as a distributed knowledge structure, identify obstacles which hinder its development and suggest how to solve this problem by means of communication and interaction. We furthermore introduce the concept of "boundary artefacts", describe their implementation as tools for artefact-mediated interaction and show how this has been implemented on a commercially available collaboration platform. The article ends with a discussion and conclusions, including limitations and implications for further research. 


\section{Literature Review}

Our research is highly innovative, in fact the aforementioned main topics, i.e. "process model of collaboration", "shared knowledge structure" and "knowledge-based collaboration" are missing in the academic and business literature on collaboration and knowledge sharing. This can easily be checked by doing an internet search as well as by analysing literature review articles. A systematic literature review of state-of-the-art functionalities in knowledge sharing mechanisms provides a representative example (Navimipour \& Charband, 2016). The research topics identified in this review have not changed since the beginning of knowledge sharing research in the 1990s: motivation for sharing knowledge, impact of good practice and job satisfaction, cultural influences, strength of social ties, explicit knowledge vs. tacit knowledge, trust, empowerment, leadership style, leadership behaviour, impact of IT (platforms, etc.), exchange relationships with co-workers, social interaction, sociocultural factors (like trust, shared goals, shared language and collaboration). Thus, the literature focuses mostly either on outcomes or on "external" aspects as conditions for successful outcomes. As a result, we can conclude that there is practically no literature about how knowledge sharing actually proceeds or the steps of its process. Notice moreover that collaboration is considered only to be a factor influencing knowledge sharing and never as a factor affected by it, like in the seminal work by Roschelle and Teasley (1995) which started the investigation into the role of knowledge sharing in collaborative problem solving in a fundamentally new way, which has never been followed up by other researchers.

Recent surveys show that, in order to cope successfully in the VUCA world, organisations should develop collaborative cultures, embrace collaborative practices (IDC, 2016, p.42) and invest in collaborative business models (Dufft, 2017, p.11). Unfortunately, the business and education world seem to largely ignore the fact that collaboration, in the wake of the spread of knowledge-intensive work (Smith, 2002; Bettoni, 2000), has also changed and is increasingly emerging as a new way of working and learning together which we call "New Collaboration". Surveys about the application of digital collaboration in business show that e-mail (which promotes divergent interaction) is still considered to be the most important collaboration tool (Roten et al., 2016, pp.3-4). But New Collaboration requires a completely different type of interaction: convergent interaction. Hence, considering and applying e-mail as the main collaboration tool clearly indicates that New Collaboration is still going unnoticed or at least is not well understood and not sufficiently put into practice.

Stoller-Schai, a pioneer of e-collaboration research, recently defined collaboration as "the direct and mutually influential active confrontation of two or more people, oriented towards common goals, to solve or master a task or problem" (Stoller-Schai, 2021). While this is very good as a generic definition, let us look more deeply at the aforementioned "task or problem". In the VUCA world, more and more groups are facing knowledge-intensive tasks which are judgmental rather than intellective (have a demonstrably correct solution), complementary (require abilities, skills, knowledge of different people) rather than conjunctive and unitary (cannot meaningfully or efficiently be divided into subtasks and assigned to different group members) rather than divisible (for these categories see: Laughlin, 2011). And for these tasks they need New Collaboration.

\section{Methodology}

To answer our main research question (how is the JKB constructed and maintained, see above), we take a Systems Thinking approach, a way of thinking which enables to better understand and design complex issues. Systems Thinking requires (Haberfellner, 2002; Vester, 2007):

1. the systemic definition of concepts. We define four new concepts in this way: a) new collaboration, b) joint knowledge base, c) artefact-mediated interaction, d) boundary artefact;

2. the use of these concepts in the context of a holistic view;

3. the use of systemic models. We describe two such models: a) distributed knowledge structure, b) bicyclic knowledge conversion;

4. the use of holistic, interconnected thinking. We have applied this in two cases: a) system of boundary artefacts, b) QUBE New Collaboration.

\section{Results}

After defining the main characteristics of New Collaboration, we specify the role of social negotiation in constructing the Joint Knowledge Base. We then model the JKB as a distributed knowledge structure, thus clarifying the issue of its existence; we identify obstacles which hinder its development and suggest how to solve this problem by means of a model which combines communication and interaction. We further introduce the 
concept of "boundary artefacts", describe their implementation as interaction tools and demonstrate how our theory works in practice by showing its implementation on a commercially available collaboration platform.

\subsection{New Collaboration: Concept, Definition, Process}

If we want to fully exploit New Collaboration, we need first of all to become aware of its emergence and secondly to better understand its structural and dynamical complexity. This requires a clarification of our concept of New Collaboration, a description of its essential elements (structure) and a model of how New Collaboration unfolds (method, process).

Successful groups are increasingly collaborating in this new way: they work together on a knowledge-intensive, judgmental and complementary task without splitting it. And because the task (the unit of work) is not split, the related knowledge needed during the performance of the task must also form a unit and be maintained as a unit. For this reason, New Collaboration requires the individual knowledge of the collaborators to be integrated into a shared knowledge structure. This essential "Rule of Unitary Group-Knowledge" can be concisely stated as:

IF the group-task is unitary, THEN group-knowledge should be unitary.

In this sense, we say that New Collaboration is knowledge-based. Moreover, the practice of constructing and maintaining this task-related knowledge as a shared knowledge structure requires that the group's members accept fair terms of collaboration, like reciprocity or mutuality (Rawls, 1971; 2001). Against this background, group collaboration has three relevant dimensions: (1) the mutual engagement of the group in a conscious, continuous effort to pursue (2) a joint enterprise (shared tasks) which, over time, leads to the development of (3) a shared repertoire (symbols, words, concepts, stories, ways of doing things, routines). Since these are the three dimensions of practice in Wenger's community of practice model (Wenger, 1998, pp.72ff), it is in this sense that we say that New Collaboration is community-oriented. The aforementioned characteristics of the concept of New Collaboration are summarised in the following definition (adapted from Bettoni et. al., 2016; Bettoni, 2017):

New Collaboration is a way of working together at a complementary and unitary task without splitting it, thereby concurrently practicing fair terms of collaboration and being mutually engaged as a community for constructing and maintaining a Joint Knowledge Base as a basis for accomplishing that task.

This definition has its roots in the seminal work by Roschelle and Teasley (1995) which investigated collaborative problem solving almost 25 years ago. In our process model "Pyramid of New Collaboration", we have modelled this type of collaboration by means of a breakdown structure similar to the "Work Breakdown Structure" (WBS) of project management; it is a hierarchical decomposition with seven layers, where each layer specifies the means that enable the process in the upper layer, and all the layers together implement the whole collaboration process. With this approach, collaboration (the highest level) is based on knowledge sharing (the $6^{\text {th }}$ level) as the essential means which enables collaboration. This aspect, new collaboration being knowledge-based, is perhaps the main difference from traditional collaboration and requires us to elaborate our concept of a JKB in more detail.

\subsection{Joint Knowledge Base}

The term Joint Knowledge Base (JKB) indicates the shared knowledge structure which is constructed and maintained during collaboration on a unitary task (new collaboration). According to Roschelle and Teasley (1995, p.76), collaborators interact through language (conversation), physical action and combinations of words and action. During these collective activities, each collaborator contributes their unique knowledge (Fig.1) to the group work and from this interactive process, shared knowledge elements emerge, the building bricks of the JKB.

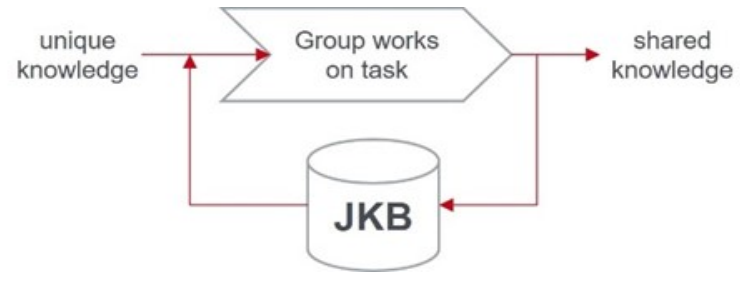

Figure 1. Group working on a unitary task - the JKB as an interaction bridge 
The JKB collects and organises these knowledge elements into a system. Thanks to this, it can function as a basis for accomplishing the shared task on which the group is working and can also be seen as a control unit regulating the interaction, a bridge between shared and unique knowledge (interaction bridge), an essential condition for the possibility of successful collaboration. In our Pyramid Model of New Collaboration (Bettoni and Obeng, 2020), Knowledge Sharing is placed immediately below it, at level six, as the fundamental activity which enables New Collaboration (the highest level). It is here where collaborators construct and maintain the Joint Knowledge Base. In order to do this and organise the knowledge elements into a system they must interact in new ways described at the underlying levels five and four, called "Negotiation of Meaning" and "Co-Construction of Knowledge" (Bettoni and Obeng, 2020, p.167).

\subsubsection{From IKB to JKB: Negotiation of Meaning}

Before entering the JKB, any knowledge element must have been previously constructed by an individual group member (collaborators) and be part of their Individual Knowledge Base (IKB). The IKB is not merely storage for knowledge elements; in line with Immanuel Kant's definition of knowledge ("A system of compared and connected mental constructs", Kant, 1781/1787, A97, own translation), we conceive it as a knowledge system which plays a role in basic cognitive functions such as assimilation and accommodation (Piaget, 1936). Thus, an IKB is much more than simply a repository and contributes a greater functionality to cognition than just a memory system. It is hence important to understand how a knowledge element of an IKB can become part of the JKB so that it can be considered as "shared". In order to be accepted as constitutive parts of the JKB, knowledge elements must be evaluated as meaningful by the group. The meaning that they must have is not simply a specific relationship between a sign and a reference (lexical meaning) or a grand principle of reason or ethics (philosophical meaning). They must make sense to the group in practical ways, especially in relation to the professional experience of each group member. This is why the knowledge elements can enter the JKB only if they successfully pass through a "sensemaking" process called a negotiation of meaning: an interactive process which comprises two highly interlinked activities, participation and reification (Wenger, 1998), and which is the first of the two main components of cognitive presence (Bettoni et al., 2018). In short: any element of an IKB of any group member must go through a process of group cognition and can enter the JKB only as a socially negotiated result of this process.

Participation must be about something, some content, ideas, proposals; and reification also must be of something. So, what we need here in first place is to produce relevant content, hence to be creative and skilled in constructing (producing or modifying) knowledge elements. Thus, the core process underlying and enabling the negotiation of meaning is the co-construction of knowledge, the second main component of cognitive presence. It comprises skills like: (a) shared language, (b) shared content / storage, (c) co-planning, (d) co-solving, (e) cowriting (Bettoni et al. 2018, p.1137).

\subsubsection{The JKB as a Distributed Knowledge Structure}

During the course of the co-construction of knowledge, each collaborator builds and maintains in their mind their own individual knowledge base so that, within a group, we have as many IKBs involved as there are collaborators. But the overall shared goal of working with the other collaborators on the same shared task leads to the emergence of areas within the individual knowledge bases which mutually converge (and resonate). When the negotiation of meaning succeeds, this leads to the emergence of knowledge areas in each individual which converge and resonate among the group members: the elements of these areas can then be considered as "shared" knowledge elements. Thus, the Joint Knowledge Base does not exist in a single place and is not constructed outside the minds of the collaborators. There is no single, integrated knowledge base that grows outside of them; instead, a JKB is a distributed knowledge structure made of the converging parts found within the individually constructed knowledge bases (Fig. 2). 


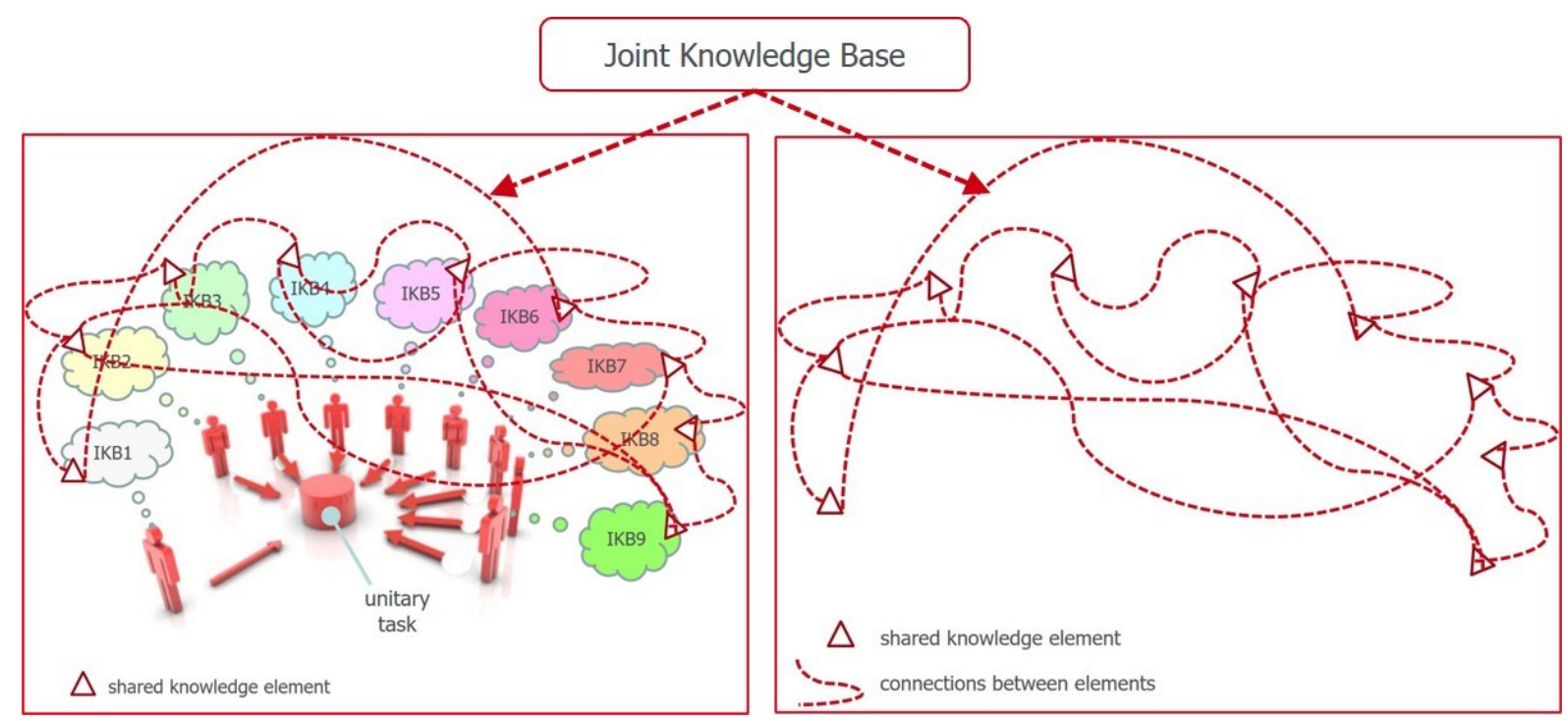

Figure 2: Joint Knowledge Base as a distributed structure (one shared element, all group members).

It is in this sense that we speak of a "joint" knowledge base: the JKB is the distributed system connecting and comparing all those individual knowledge elements whose meanings converge and fit across group activities. Thanks to this, the JKB enables meaningful conversation, action and interaction in relation to the purposes which emerge, step by step, during collaboration on a shared task. Any JKB is bound to the specific group of individuals who interact: when one individual is missing or a new one arrives, then the JKB changes. The fact that two or more individual knowledge elements are seen as converging does not necessarily imply that they precisely overlap or match in all their parts and across all the individual knowledge bases of the group members. It is sufficient if they are fit for the purposes in hand. In this sense, we should speak more precisely of taken-asshared rather than shared knowledge elements (Cobb, 2000, p.166).

\subsubsection{Knowledge Sharing Obstacles}

When collaborating in this way, the JKB can grow and step by step become more and more representative of the group's knowledge. But due to the complexity of communication and interaction, co-constructing such a representative JKB is not easy to achieve! When people engage in conversations and debates for contributing knowledge elements and negotiating their meaning, they will encounter many obstacles. Two of the main obstacles (also called "semantic boundary", Carlyle, 2002) are:

1. the meaning of messages cannot be just transferred by communication;

2. interpretations of messages are often different.

The first obstacle exists because meaning does not travel; it must be (and always is) actively constructed. Heinz von Förster called it The Hermeneutic Principle: "The hearer, not the speaker, determines the meaning of an utterance" (Förster and Glasersfeld, 1999, p.13). The second obstacle exists because differences in experience and background among participants have created different experiential worlds (Glasersfeld, 1991, 2007) and these lead to different conceptualisations and interpretations which, on one hand, may be a source of creativity but on the other, can create social dilemmas and conflicts (Du Chatenier et al., 2009).

These intrinsic obstacles allow us to recognise something very important for collaboration: that plain conversation is an intrinsically limited resource when it comes to constructing a JKB. We know this very well from conventional meetings: they are very often not only unproductive and a waste of time (Kello, 2015) but also last too long, mostly because the collaboration and knowledge sharing are not working as they could and should when the meeting only uses plain conversation and debate (Bettoni and Obeng, 2020). Thus, as a next step, we need to solve this problem with the intrinsic limits of conversation.

\subsubsection{Bicyclic Knowledge Conversion}

Conversation is a complex process, but since language is learned without effort (in childhood), that process unfolds hidden behind a "veil of simplicity" (in analogy to the "veil of ignorance", Rawls, 2001), that we put on step by step during the course of our language skills development, until we reach the highest level, that of unconscious competence (Flower, 1999). By looking behind this veil, we can see how, during conversation, a 
knowledge base is built and maintained by several element-related activities which are performed on the knowledge elements during a circular, recursive process.

The four main activities are:

1. introducing new elements (through assimilation or accommodation);

2. modifying existing elements when divergence arises during collaboration;

3. detecting divergence across collaborators by monitoring ongoing interpretations of knowledge elements and comparing them with the intended interpretations to determine whether these fit;

4. last but not least, rectifying intended interpretations when there are conflicts (meanings do not fit).

If we want to solve the aforementioned problem of the intrinsic limits of conversation, then the complexity of communication and interaction involved in such knowledge sharing activities should be taken seriously. To this aim, we have developed a model of bicyclic knowledge conversion between a subjective self and another person, indicated by "Self" and "Other" (Fischer and Herr, 2019). The model is called "bicyclic" because it combines two main ways of recursive, coordinated interacting (Fig. 3): a communication (lower half of the diagram, based on Glanville, 2007) and a co-action (upper half of the diagram).

Since we focus on knowledge conversion in our model, in constructing it we used the SECI model of knowledge conversion (Nonaka and Takeuchi, 1995) and the IECS basic knowledge sharing model (Kharabsheh et al., 2016) as sources of inspiration. But differing from the $\mathrm{SECl}$ model, in our model the interaction process begins by explicitly considering two individuals, each with their own individual knowledge base (explicit and tacit knowledge) and looks at how knowledge conversions proceed first within each single person and then between them. More precisely, typical bicyclic knowledge conversion starts within the individual and proceeds as follows (Fig. 3, left side):

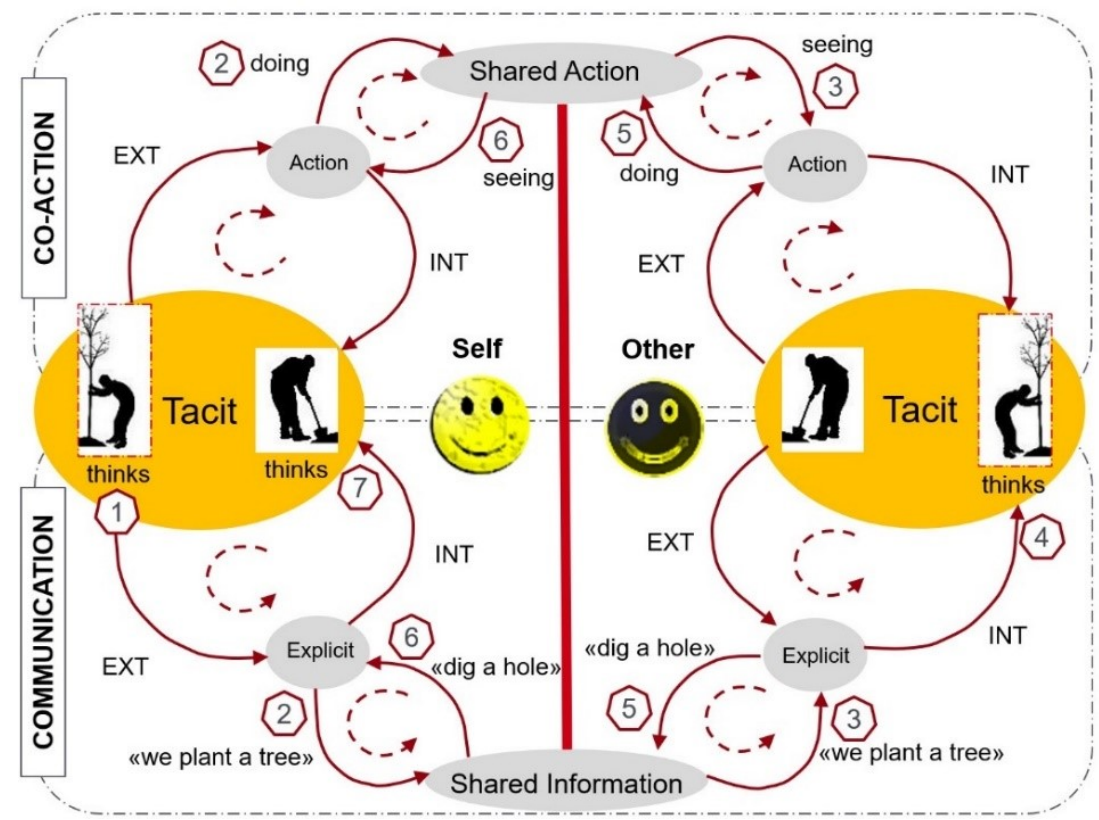

Figure 3: Bicyclic knowledge conversion between the Self and Other.

1. First, the model considers two knowledge conversions by the Self, from tacit to explicit (acronym EXT) and from explicit to tacit (acronym INT). EXT means "knowledge externalisation" (to document, to express) whereas INT means "internalisation" (to learn, to understand).

2. These two knowledge conversions form a loop internal to both individuals, indicated in the diagram with a circular arrow (dashed line). This loop can eventually lead to some thoughts related, for example, to planting a tree, indicated in the diagram - within the tacit knowledge base - by the silhouette of a person holding a young tree.

3. In a third step, the model looks at the knowledge conversion from explicit to explicit called "combination", which in our case is seen as an exchange of information in the sense of a conversation between two individuals. 
4. At the same time, we also look at knowledge conversion from tacit to action, depicted in the upper part of the diagram.

5. An interactive knowledge conversion between Self and Other begins with the conversation depicted in the lower part of the diagram but is interlinked with the process of co-action.

- Step 1: Mrs Self thinks of planting a tree.

- Step 2: She says "we plant a tree" and, at the same time, the action of catching, moving and holding the tree begins.

- Step 3: Mr Other hears "we plant a tree"; this is the only information transferred. Meaning is not transferred, so what does this sequence of words mean? If there were no action, interpretation could be difficult and lead to misunderstandings. But Mr Other sees what Mrs Self is doing.

- Step 4: He understands correctly what Mrs Self said and contributes a complementary idea, for example by thinking that the tree needs a hole.

- Step 5: He says "dig a hole" and begins to do it.

- Step 6: Mrs Self hears "dig a hole". Again, if there were no action, interpretation could be difficult. But Mrs Self also sees what Mr Other is doing.

- Step 7: She understands correctly what Mr Other said and contributes a complementary idea, for example by thinking of a combination of her holding the tree and Mr Other digging a hole: they fit and so the collaboration is successful.

We see here that, thanks to co-action, interpretations on both sides were correct and conversation has been successful; moreover, thanks to this intertwining of conversation and co-action, a fourth step of knowledge conversion called "socialisation" has also been successfully performed; this is the step in which tacit knowledge is shared between the two individuals by means of integrating the two types of interaction, conversation and physical action. This suggests that the problem with the intrinsic limits of conversation can be solved if the group collaborates (and constructs the JKB) in a way so as to intentionally make use of both conversation and co-action.

\subsection{Artefact-Mediated Interaction with Boundary Objects}

Given the previously presented model of a JKB, our next task now consists in suggesting collaborative patterns for group work and business meetings which promote this way of collaborating. Facilitation techniques already answered this question in part a long time ago by adding a specific type of physical action in space to conversation. This approach increases the complexity of leading the interaction, but we know from the practice of facilitation (Wagenaar and Hulsebosch, 2008; Kaner, 2014) that there are lot of tools and techniques (which can be easily learned) which help skilled facilitators in coping with this complexity of leading. Complex interaction methods, if appropriately facilitated, best fit with the complexity of the co-construction process, thus enabling the group to interact in a much more efficient and much more effective way.

A knowledge café, which requires dialogue rules, a specific venue with tables (each for 4 or 5 people), a facilitator with the skills to chair the session, a powerful question, a relevant introduction, small group conversations, etc. is a good example of such a complex interaction method (Sharp, 2019; Gurteen, 2021). Physical action is implemented mainly by means of the session's assembly changes (from plenary to small groups, from group to group 3 times then back to plenary) but can also include notes taken by all participants on large shared sheets at the tables.

Another example is storytelling: in this method, the physical action comes with the chain and flow of events which make up the story; we find it in the central conflict of the story as well as in its resolution. What we have here is merely a described and imagined physical action but it is there as a new dimension which increases the opportunities for activating the imagination, stimulating further thinking, creating tacit knowledge, helping to rediscover meaningfulness and orientation, awakening emotions, creating cohesion and strengthening memories. Among other types of stories, fairy-tales are particularly suited to fostering collaboration because they bring and hold together individuals and their (tacit) experiences through references, metaphors and analogies which can easily be assimilated (Bittel and Bettoni, 2014).

\subsubsection{Artefact-Mediated Interaction}

When conversation and physical action on an object (in space) are combined into one single group activity, we obtain a new type of interaction, which we call object-mediated interaction (combinations of words and actions on a shared object), where the physical action is performed on real objects which can be created, moved, 
modified and transformed in various ways in space. The new dimension which physical action adds to conversation and the object which is produced step by step over the course of the interaction largely increase the number of choices and hence the opportunities for knowledge co-creation.

When the object is an artefact produced by the group (like, for instance, a list of ideas, a mind map of concepts or a flowchart of a process) and this artefact is made easily visible and accessible for everybody by means of a poster, a whiteboard, a panel or a table, then we speak of artefact-mediated interaction. In artefact-mediated interaction, the physical action performed by the collaborators consists of moving within a shared 3D space, sitting in small groups at tables, gathering in-front of a panel or poster, writing on large sheets or on small cards, fixing them on a panel or wall and then organising them according to a given problem-solving method etc. In doing so, they not only use existing artefacts; they also change or produce one or more of them as a result of their interaction. Such a product could be a set of marketing ideas organised in subgroups, a form for systematically organising information about project progress or a plan of action for training new clients.

When studying collaborative, heterogeneous problem-solving by scientists from different disciplines, Star (1989) recognised the importance of such collaboratively-produced artefacts. In fact, she observed that those scientists, despite their differences, were nonetheless able to successfully collaborate and attributed this to what she called "boundary objects": objects produced during the interaction that enabled those scientists to establish shared context (Star, 1989, p.47) like, for instance, documents, terms, standardised forms and methods, models, maps and other forms of reification (Wenger, 1998, p.105). In order to underline the fact that these objects are produced during the interaction, we will call them "boundary artefacts". They can link communities together by allowing different groups to collaborate on a common task (Wenger, 1998, pp.105ff) but they can also link individuals together by allowing a group to collaborate on a unitary task.

\subsubsection{A System of Boundary Artefacts}

Although traditional facilitation methods and tools (Kaner, 2014) use boundary artefacts, this alone is not enough to cope with the complexity of the co-construction process and to produce a representative JKB. We need a more systematic approach, which identifies a set of relevant dimensions (like space, time, culture, tools, methods and behaviours, see section 5) and where the whole interaction process is designed around boundary artefacts. Implementation of such a systematic approach exists in the QUBE system, a 3D collaborative virtual environment (3D CVE) of the "virtual world" type (Pentacle, 2015).

On QUBE, a boundary artefact is called PET (abbreviation for Performance Enhancing Tool). A QUBE PET implements a set of knowledge elements on a pinboard, structured in a specific way (see Fig. 4 and 5): this given structure functions as catalyser for the interaction, thus guiding the construction of shared knowledge elements along clearly defined pathways and making the negotiation of meaning more efficient and effective. Four of the most successful QUBE PETs are Hopes\&Fears ${ }^{\mathrm{TM}}, 5 \mathrm{Ps}^{\mathrm{TM}}$, StickySteps ${ }^{\mathrm{TM}}$ and RAPID ${ }^{\mathrm{TM}}$ :

- $\quad$ The PET called Hopes\&Fears ${ }^{T M}$ (Fig. 4) has a pinboard divided in three fields: on the right, a box for the fears, on the left a box for the hopes and below, a box for ways for overcoming the fears. This is a great way to initiate any new activity with stakeholders, especially if there is lots of uncertainty in the goals or methods. Working with this PET helps with the first steps of inclusion in teambuilding; it contributes to emotionally engaging team members from the start, helping them to define shared goals, agreeing early on what does and does not fall within the scope, agreeing on what are hard and what are soft success criteria, identifying risks and creating the basis for a common project culture.

- The PET called 5Ps ${ }^{T M}$ (Fig. 4) provides a way of formulating the essential aspects of a message, task or action. The pinboard is divided in two fields. The box on the left is for the unstructured, spontaneous description of an idea, message or task. The box on the right is structured into 5 fields: Purpose, Principles, People, Process and Performance. Working with this PET helps to avoid misunderstandings and communicate clearly about complex issues, like the core elements of a project, problem or solution. It should be used at milestones when the group needs to reflect on the project, task or action and gain a clearer view of its essential aspects as well as every time a team member or stakeholder receives instructions to act independently. 

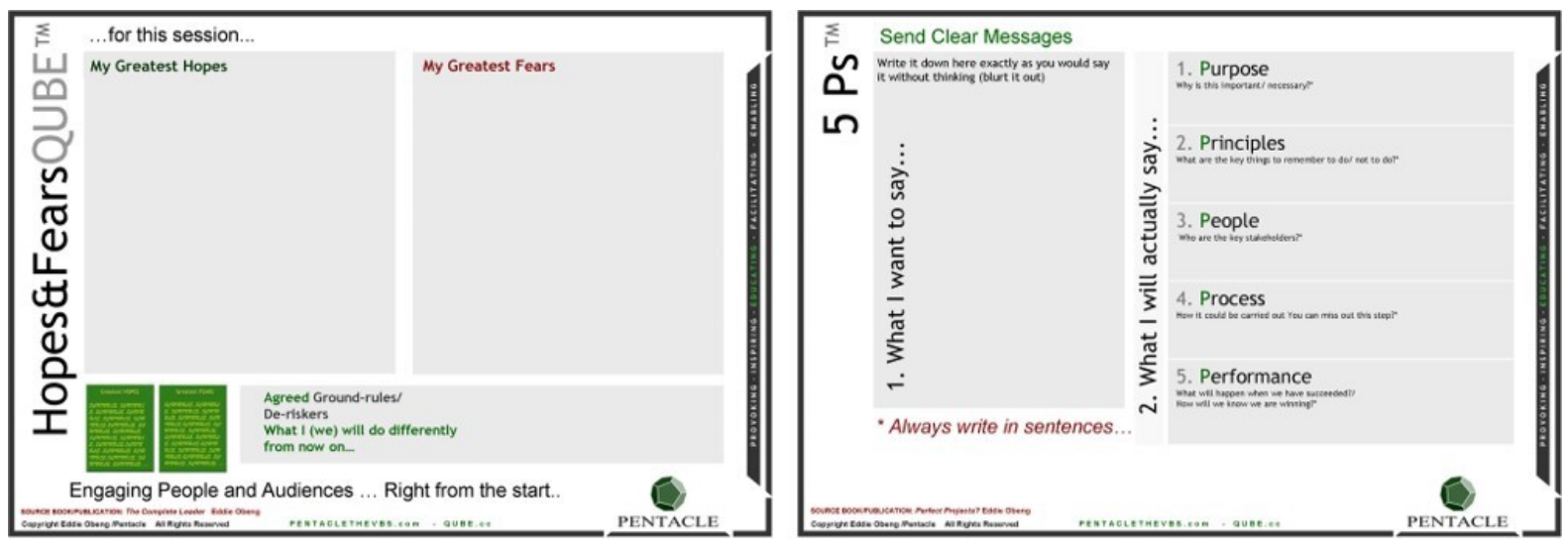

Figure 4: Boundary objects Hopes\&Fears ${ }^{\mathrm{TM}}$ and 5Ps ${ }^{\mathrm{TM}}$ (www.pentacle.co.uk).
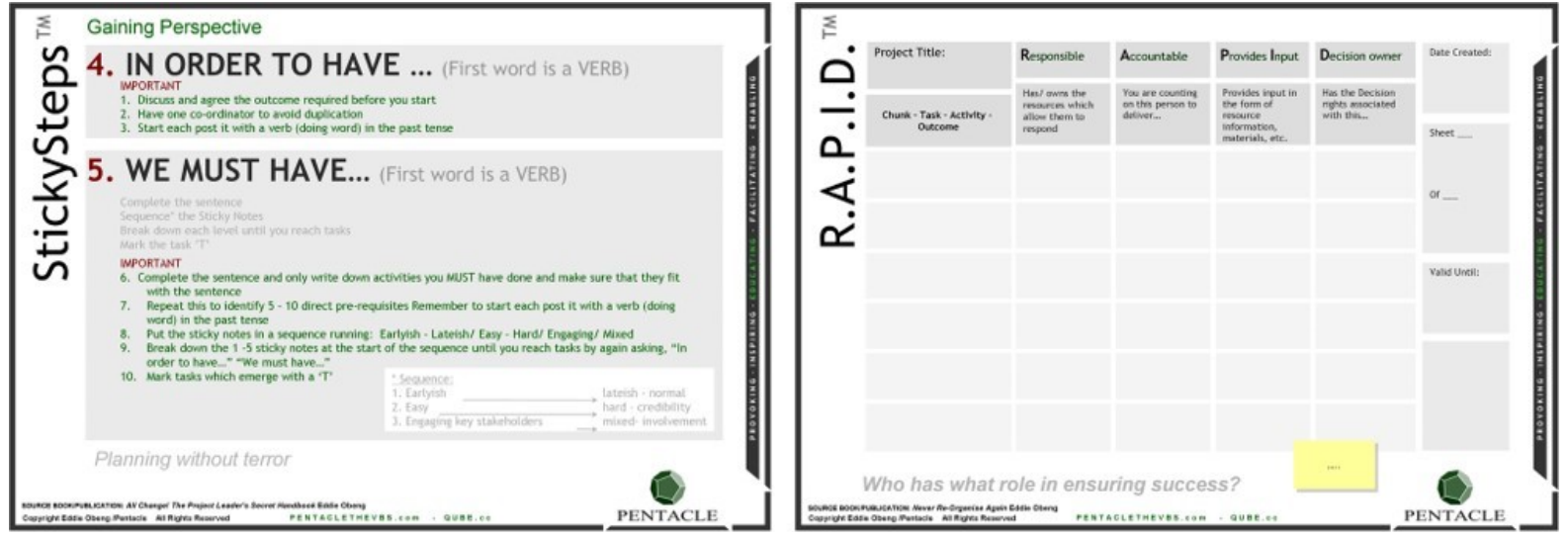

Figure 5: Boundary objects StickySteps ${ }^{\mathrm{TM}}$ and RAPID ${ }^{\mathrm{TM}}$ (www.pentacle.co.uk).

- $\quad$ The PET called StickySteps ${ }^{T M}$ (Fig. 5) displays a simple pinboard with just two areas; a row on the top for expressing the overall goal and a large area below this row for breaking down the overall goal of the action into the means required to achieve it. If one or more actions in this lower area are still too large, the team repeats this step by adding a lower breakdown level; the breakdown continues until you reach tiny little steps (tasks). This PET provides a quick and effective way to plan a project or action whenever they are almost too large to contemplate. This kind of breakdown (goal to action) allows interdependencies between parts of the project, action or task to be dealt with appropriately.

- The PET called RAPID ${ }^{T M}$ (Fig. 5) is simply a table of tasks (rows) with columns for the different roles (responsible, accountable, contributing and deciding) needed for accomplishing a task; when roles for tasks are assigned to group members, then the facilitator enters their names in the related cells, thus making sure that everyone knows exactly what it is they have to contribute, and in what way. This PET is also very useful for ensuring the interdependence of the activities and people involved on them in a systematic way: this helps to reduce bottlenecks which tend to slow down the pace of execution of a project. It should be used at the end of any team meeting and in general every time the group agrees on tasks for a piece of work in which the group members are involved.

\subsection{QUBE New Collaboration}

On QUBE, the previously mentioned systematic approach to online collaboration is obtained by designing a meeting around six relevant dimensions, which can also be seen as six relevant components: Physical Space, Time, New Culture, New Tools, New Methods, New Behaviours. Strictly speaking, artefacts-mediated interaction is implemented by means of the $5^{\text {th }}$ component (New Methods) but all the other dimensions also contribute and are necessary for the QUBE New Collaboration:

1. Physical Space on QUBE is supported by a 3D environment (3D CVE) which simulates a business campus with buildings, various types of rooms and other places and provides avatars with which each participant can move around in the rooms, meet other people, sit with them and chat at tables, visit panels, point to items on the walls of the room, walk to other rooms, work at an individual desk, etc. 
2. Regarding Time, on QUBE a meeting continuously uses boundary artefacts, in each phase, from begin to end.

3. An example of "New Culture" is the principle of "Write first, talk second" which requires that, for any given topic of discussion, participants must first write their contributions on a panel and then conversation will start based on and referencing those written contributions.

4. An online "New Tool" is, for example, the sticky note facility and the panel on a wall: each participant can create as many sticky notes as they wish, write a text on each and place the sticky note on a panel visible to all the other participants.

5. A "New Method" is, for instance, the so-called "Hopes \& Fears" method for collaboratively planning a session.

6. Last but not least, an example of a "New Behaviour" is being fully attentive to what is going on instead of reading e-mails or surfing the internet during the meeting.

Thus, in general, on QUBE, collaboration and the construction of a JKB proceed as a group activity where cognitive, social and leading presence come together at the intersection of these different dimensions and produce boundary artefacts.

\subsubsection{A QUBE Session}

A typical session on QUBE, for instance a kick-off meeting, begins with session facilitators welcoming the participants as they arrive in the space. Each person in QUBE is represented by an individual avatar, a simple box figure (like LEGO mini figures but gender-neutral) which provides enough of a human form to foster the required level of identification.

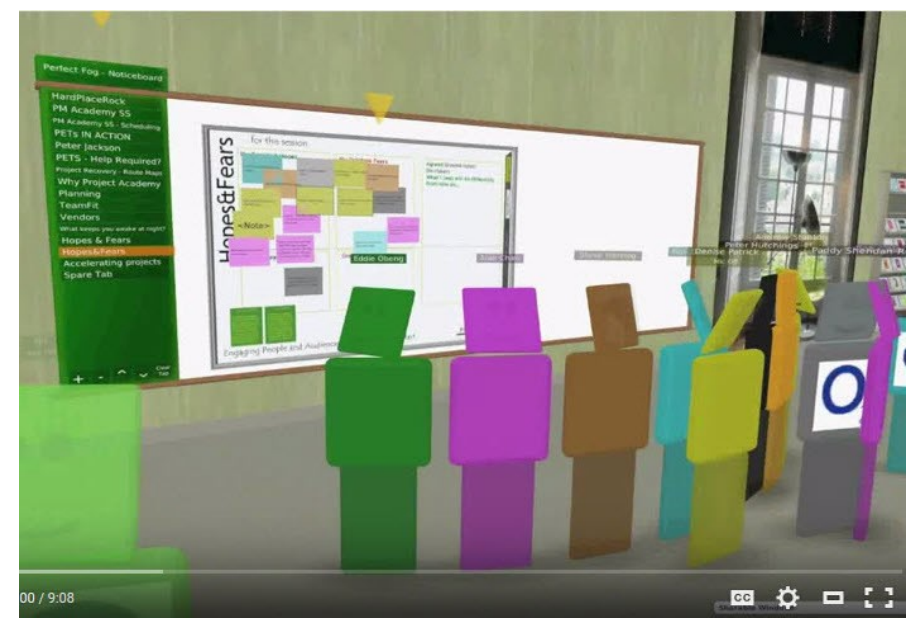

Figure 6: Group at whiteboard with Hopes \& Fears PET by Pentacle (http://www.pentacle.co.uk/)

Using your avatar, you are able to communicate with other people just as you would in the real world. You can move around in the rooms of a building, physically interact and work shoulder to shoulder, literally brainstorming with many other people by means of whiteboards and sticky notes.

The facilitators welcome each one individually and make sure that they are ready to begin. Then the avatars can visit the virtual collaboration space until the meeting starts. The room has been carefully prepared in advance. The whiteboards, panels, tables and chairs needed during the meeting are available on the walls and on the floor. Some whiteboards display poster tools called PETs (Performance Enhancing Tools), guidelines or procedures about how to accomplish a task described on a poster. PETs can easily be replicated on a whiteboard or wall when needed; each PET is linked with a specific documentation which describes "what is it?", "why do I need it?", "when do I use it?" and "how do I use it?" In this sense, a QUBE PET is a collaboration method and corresponds to what is often called a collaboration pattern in literature (Eppler and Schmeil, 2010). What is special about a QUBE PET is that the collaboration involved is always an artefact-mediated interaction.

\subsubsection{Problem-Solving Meetings}

Regularly scheduled problem-solving and decision-making meetings with a project team are the most important requirement for effective and efficient collaboration (Gordon, 1977). On QUBE, these meetings, called 
"drumbeats", eventually receive the high level of consideration which they deserve. Within such a drumbeat, we see exemplified how QUBE implements artefacts-mediated interaction: conversation and physical action are combined during a meeting on QUBE to form a mediated interaction in which collaborators produce the aforementioned boundary artefacts (see 4.3.1). A drumbeat starts with a previously appointed coordinator welcoming the team members as they arrive in the project space on QUBE and gather at the central meeting place, called the "home" (leadership presence, social presence). When the group is complete, they walk together to a panel which displays the PET "Hopes \& Fears" where the coordinator asks: "what are your expectations and what are you afraid of in relation to this meeting?". Each participant writes their contributions on cards and places them in the hopes or fears area of the panel (Fig. 4, left). Collaboration enabled by this boundary artefact starts within the individual, with the two knowledge conversions of "internalisation" (INT) - when a participant reads the cards that other participants place on the board - and "externalisation" (EXT) - when a participant writes their own contributions on cards and places them on the board, among the others. A team member will then organise the cards in clusters and clarify their meaning with the help of the group, thus beginning the knowledge conversion of "combination". Together the team then needs to discuss what could remove the Fears (social presence) before creating a meeting agenda of steps for making the Hopes a reality (cognitive presence): this will require writing some new cards and placing them on the board as well as putting existing cards in a proper temporal sequence. The knowledge conversion of "socialisation" happens in these card-related actions: pointing to a card when talking about its content, moving cards to put them in a better order, creating clusters or sequences of cards, changing the size of a card, formatting its words, etc.

When the meeting agenda is ready, then the team starts working on the tasks defined in it. PETs like " 5 Ps" and "STICKY STEPS" help to clarify the problem and plan some quick wins in the beginning. During the interactions, some specific questions will arise and provide opportunities for starting work in smaller groups. Small groups can move to an area in the same room equipped with chairs and round tables and sit down here when they want to discuss something, for example how to proceed when dealing with the specific question they have selected to work on (cognitive presence, leading presence). Once they have decided this, they can move to another area of the space and gather in front of a huge whiteboard with sections separated by panels. At tables and in front of panels, the group members will only hear each other talking, without noise from other groups (a feature which is quite impossible in a real room). At the end of the meeting, a PET called RAPID will help the whole team to define the next steps and related tasks and plan when and who will accomplish them after the meeting (leading presence, social presence). After that, the team usually gathers in a circle in the middle of the room. Here the group performs a so-called "spincasting" (social presence): each team member in turn has the opportunity to give brief feedback about the work carried out in the small groups (insight, remarks, questions, learning, etc.).

This structure of a drumbeat meeting in three steps (start in plenary with a teambuilding PET, work in small groups with problem solving and planning PETs, closing the meeting with a planning PET and a plenary feedback) can also be applied during any phase of project work.

\section{Discussion}

In the VUCA world, the trend is clearly moving towards increasingly knowledge-based work in a fast-changing environment. In order to successfully cope with this evolution, organisations should develop collaborative cultures and embrace collaborative practices. New Collaboration can support this but in order to fully exploit its potential, we need to better understand an essential aspect of knowledge sharing, the Joint Knowledge Base.

To this aim, we have first provided a definition of New Collaboration as a knowledge-based and communityoriented activity where collaborators are mutually engaged in constructing and maintaining a JKB as a basis for accomplishing their unitary task without splitting it. At first sight, this concept of collaboration may seem excessively resource-intensive; imagine, for instance, a research department; what could it mean here, "working together on a unitary task without splitting it"? Of course, researchers should not work all together on every research project and on every work package in a project. There are tasks which were, are and will also in future be better assigned to one individual. But the trend is clearly moving towards increasingly knowledge-based work units (Jacobs, 2019) and because knowledge evolves so rapidly and remains mostly tacit (less time for documenting) due to the high rate of innovation, these tasks tend to be complementary (require the contribution of more than one individual). When problem-solving requires interaction with other people, what you have is often a consulting situation. Sometimes just a phone call or e-mail to one colleague may be enough; 
but in many cases, you need more time and to involve more than one person. You may need a prolonged, problem-solving interaction with a group and this can seldom be done one-to-one on the phone; it requires a meeting. So, with increasingly knowledge-based, complementary and unitary tasks, the number of (problemsolving) meetings is also increasing. These meetings are critical for any organisation, but most people hate them (so-called "paradox of meetings"). Fortunately, New Collaboration also applies to problem-solving meetings and has recently been proposed as a solution to the paradox of meetings (Bettoni and Obeng, 2020).

The knowledge elements of a JKB are all initially constructed by individual group members and must then go through a process of group cognition (a negotiation of meaning): they can enter the JKB only as a socially negotiated result of this process. This approach implies that the group must pay great attention to the two components of the negotiation process, participation and reification.

By arguing that participation and reification are both based on and enabled by the co-construction of knowledge, we have in a certain sense shifted the focus to the intrinsic limits of plain conversation as a method for building a JKB, like for instance in conventional meetings. Our model of bicyclic knowledge conversion has enabled us to look behind the veil of simplicity: there we were able to see not only the obstacles which hinder and the activities which contribute to the construction of a representative JKB but also to understand the essential role that physical action can play in overcoming the problem of the intrinsic limits of plain conversation. Based on this, we have proposed artefact-mediated interaction as a solution to this problem and argued for the need of a systematic approach, where the whole interaction process in all its relevant dimensions (like space, time, culture, tools, methods and behaviours) is designed around boundary artefacts. Finally, we were able to show that this way of collaborating is not just theory but already daily practice: the commercially available collaboration platform called QUBE and the tools and culture which it promotes constitute a real-world system which successfully implements artefacts-mediated interaction by means of boundary artefacts (PETs), thus enabling the Joint Knowledge Base to be representative of the group's knowledge and the full potential of New Collaboration to be exploited.

\section{Conclusion}

Our paper deals with the fundamental issue of a Joint Knowledge Base, which so far was missing in the research literature. Our model of a JKB adds a missing piece to the description and generation of collaborative group interaction patterns (Eppler and Schmeil, 2010). We believe our model to contribute an important aspect to the effort of formalizing collaboration both in virtual and in physical environments, a task that is crucial especially in order to advance the application of digital collaboration. Our research contributes further to a better development of collaboration patterns as "recommendations for how (or how not) to interact" (Wasson and Mørch, 2000), which is essential for promoting innovative, valuable new forms of working together (New Collaboration). Nevertheless, being this the first attempt to address fundamental issues like how a JKB enables collaboration and specifically how a JKB is constructed and maintained, the current version of this attempt has some limitations, which will need to be well investigated in the evaluation of this research and addressed by future work.

First of all, our model of a Joint Knowledge Base is only a rough sketch; further research will be required for investigating: 1) how knowledge areas, which mutually converge and resonate, emerge within many individual knowledge bases; 2) to what degree the meanings need to be consensual and build convergent areas in order to enter the JKB; 3 ) what indicates the convergence of meanings and how does resonance manifest itself in these convergent areas when meanings do not overlap or match across all the individual knowledge bases of the group members. Secondly, in mediated interaction, further investigations will have to deal with our interpretation and use of the boundary artefact and clarify how cognitive, social and leading presence come together and contribute to its production. In particular, regarding physical action, we need to develop a model: 1) of what this type of action adds to the interaction when it is performed on cards which can be placed and grouped on a whiteboard; 2) of how physical action influences the motivations, roles and relationships of the participants. Last but not least, the way in which the intertwining of communication and co-action contributes to the knowledge conversion called "socialisation" will require further clarification in future work. This will be important to make it easier to share tacit knowledge in a variety of collaborative situations.

\section{References}

Bettoni, M., 2000. Meeting the Challenge of Knowledge-Intensive Automation. Presentation at CAWorld 2000, 
Neugents Session, New Orleans, USA, 10 April 2000. [online] Available at: http://www.weknow.ch/marco/A2000/CAWrld/index.htm [Accessed 22 June 2021]

Bettoni, M., Bittel, N., Bernhard W. \& Mirata, V., 2016. eSF - An E-Collaboration System for Knowledge Workers. In: A. Kok \& H. Lee, eds. 2016. Cultural, Behavioral, and Social Considerations in Electronic Collaboration. Hershey, USA: IGI Global, Chapter 8, pp.157-172.

Bettoni, M., Bernhard, W., Bittel, N. \& and Mirata, V., 2018. The Art of New Collaboration: Three Secrets. In: E. Bolisani, E. Di Maria, \& E. Scarso, eds. 2018. 19th European Conference on Knowledge Management, ECKM 2018, University of Padua, Italy, 6-7 September 2018, Vol. 2, pp.1133-1141.

Bettoni, M. and Obeng, E., 2020. Alive by Meeting: A Solution to the Paradox of Meetings by means of the Pyramid of New Collaboration. Electronic Journal of Knowledge Management, [e-journal] 18(2), pp.161-171. DOI: https://doi.org/10.34190/EJKM.18.02.006

Bittel, M. and Bettoni, M., 2014. Learning CSCW through Fairytales: A Practical Model. In: A. Cercone, D. Persico, S. Fernandes, A. Garcia-Perez, P. Katsaros, S.A. Shaikh, and I. Stamelos, eds. 2014. Information Technology and Open Source: Applications for Education, Innovation, and Sustainability. SEFM 2012 Satellite Events, InSuEdu, MoKMaDS, and OpenCert. Thessaloniki, Greece, 1-2 October 2012, Revised Selected Papers. Berlin: Springer. pp.78-88.

Carlile, P.R., 2002. A pragmatic view of knowledge and boundaries: boundary objects in new product development, Organization Science, 13(4). pp. 442-455.

Cobb, P., 2000. Constructivism in Social Context. In: Leslie P. Steffe, and Patrick W. Thompson, eds. 2000. Radical Constructivism in Action: Building on the Pioneering Work of Ernst von Glasersfeld. London: RoutledgeFalmer. pp.152-178.

Du Chatenier, E. Verstegen, J.A.A.M., Biemans, H.J.A., Mulder, M. and Omta, O., 2009. The Challenges of Collaborative Knowledge Creation in Open Innovation Teams. Human Resource Development Review, 8(3). pp.350-381.

Dufft, N., 2017. Die digitalen Trends 2017: Von der Vision zur Realität! Munich: Pierre Audoin Consultants (PAC). [online] Available at: <https://www.swisscom.ch/de/business/enterprise/downloads/digitalisierung/digitale-trends2017.html> [Accessed 30 March 2020].

Eppler, M.J. and Schmeil, A., 2010. Visual Collaboration and Learning Patterns in 3D Environments: Emergence, Elements, Examples. In: T. Hug and R. Maier, eds. 2010. Medien - Wissen - Bildung Explorationen visualisierter und kollaborativer Wissensräume. Innsbruck: Innsbruck University Press.

Fischer, T. and Herr Ch. M., 2019. An Introduction to Design Cybernetics. In: T. Fischer and Ch. M. Herr, eds. 2019. Design Cybernetics: Navigating the New. Cham (CH): Springer Nature Switzerland AG. pp.1-22.

Flower, J., 1999. In the mush. Physician Executive, 25 (1). pp. 64-66.

Förster, H. von and Glasersfeld, E. von, 1999. Wie wir uns erfinden. Eine Autobiographie des Radikalen Konstruktivismus. Heidelberg: Carl-Auer-Systeme Verlag.

Glanville, R., 2007. Grounding Difference. In: Müller, A. and Müller, K. H., eds. 2007. An Unfinished Revolution? Heinz von Foerster and the Biological Computer Laboratory 1958-1976. Vienna: edition echoraum. pp. 361- 406.

Glasersfeld, E. von, 1991. Knowing without Metaphysics: Aspects of the Radical Constructivist Position. In: Steier F., ed., 1991. Research and Reflexivity. London: Sage. pp.12-29.

Glasersfeld, E. von, 2007. The Constructivist View of Communication. In: A. Müller, \& K. H. Müller, eds. An unfinished revolution? Heinz von Foerster and the Biological Computer Laboratory 1958-1976. Vienna: edition echoraum. pp. 351-360.

Gordon, T., 1977. Leader Effectiveness Training, L.E.T. - Proven skills for leading today's business into tomorrow. New York: Penguin Putnam.

Gurteen, D., 2021. The Gurteen Knowledge Café: Knowledge Café Tipsheet. Available at: <http://knowledge.cafe/> [Accessed on $17^{\text {th }}$ April 2021].

Haberfellner, R. et al., 2002. Systems Engineering, Methodik und Praxis. Zürich: Verlag Industrielle Organisation, 11. Auflage.

IDC, 2016. Future People: Workplace Evolution in the Age of Digital Transformation. [pdf] Framingham (MA): International Data Corporation (IDC). Available at <http://www.cornerstoneondemand.co.uk> [Accessed 30 March 2020].

Jacobs, R. L., 2019. Work Analysis in the Knowledge Economy: Documenting What People Do in the Workplace for Human Resource Development. Cham (CH): Palgrave Macmillan.

Kaner, S., 2014. Facilitator's Guide to Participatory Decision-Making. San Francisco: Jossey-Bass.

Kant, I., 1781/1787. Critique of pure reason. Translated by N.K. Smith, 1965. New York: St. Martin's.

Kello, J. E., 2015. The Science and Practice of Workplace Meetings. In: J. A. Allen, N. Lehmann-Willenbrock and S. G. Rogelberg, eds. 2015. The Cambridge Handbook of Meeting Science. Cambridge: Cambridge University Press.

Kharabsheh, R., Bittel, N., Elnsour W., Bettoni, M. and Bernhard, W., 2016. A Comprehensive Model of Knowledge Sharing. In: S. Moffett \& B. Galbraith, eds. 2016. 17th European Conference on Knowledge Management, ECKM 2016, Ulster University, Northern Ireland UK, 1-2 September 2016, pp.455-461.

Laughlin, P.R., 2011. Group problem solving. Princeton: Princeton University Press.

Navimipour, N.J. and Charband, Y., 2016. Knowledge sharing mechanisms and techniques in project teams: Literature review, classification, and current trends. Comput. Hum. Behav. 2016, 62, pp. 730-742.

Nonaka, I. and Takeuchi, H., 1995. The Knowledge Creating Company: How Japanese Companies Create the Dynamics of Innovation. New York: Oxford University Press.

Obeng, E., 1997. New Rules for a New World. Oxford (UK): Capstone. 
Paavola, S., Lipponen, L. and Hakkarainen, K., 2004. Models of Innovative Knowledge Communities and Three Metaphors of Learning. Review of Educational Research, Winter 2004, 74(4), pp.557-576.

Pentacle, 2015. Frequently Asked Commercial, User Concern and Technical Questions. [pdf] Pentacle, The Virtual Business School. Available at: <https://pentacle.co.uk/Downloads/DueDiligenceFAQSummary.pdf> [Accessed 22 June 2021].

Piaget, J., 1936. La naissance de l'intelligence chez l'enfant. Neuchâtel: Delachaux et Niestlé, 9th edition 1977.

Rawls, J., 1971. A Theory of Justice. Cambridge (MA): Belknap Press.

Rawls, J., 2001. Justice as Fairness: A Restatement. Cambridge (MA): Belknap Press.

Roschelle, J., and Teasley, S. D., 1995. The Construction of Shared Knowledge in Collaborative Problem Solving. In: C. E. O’Malley, ed. 1995. Computer-Supported Collaborative Learning. Berlin: Springer-Verlag. pp.69-197.

Roten, O., Zurbriggen, R. and Müller Vasquez Callo, R., 2016. E-Collaboration 2016 in der Schweiz. Bern: Sieber \& Partners AG. [pdf] Available at: http://www.sieberpartners.com/s/sp-cno-2016_E-Collaboration-2016-v10.pdf [Accessed 23 March 2020].

Sharp, P., 2019. Why Knowledge Cafés can be Valuable to Organisations. Regent's Working Papers 2019, Working Paper 1904: RWP1904. London: Regent's University.

Smith, K.H., 2002. What is the 'Knowledge Economy'? Knowledge Intensity and Distributed Knowledge Bases. Maastricht: United Nations University, Institute for New Technologies (INTECH), Discussion Paper \#2002-6.

Star, S. L., 1989. The structure of ill-structured solutions: Boundary objects and heterogeneous distributed

problem solving. In: M. Huhns and L. Gasser, eds. 1989. Readings in Distributed Artificial Intelligence. Menlo Park, CA: Morgan Kaufman, Chapter 2, pp.37-54.

Stoller-Schai, D., 2021. Making Collaboration Work. In: S. Güldenberg et al., eds. 2021. Managing Work in the Digital Economy, Future of Business and Finance. Cham $(\mathrm{CH})$ : Springer Nature Switzerland AG.

Wagenaar, S. and Hulsebosch, J., 2008. From 'a meeting' to 'a learning community'. Group Facilitation: A Research \& Applications Journal. 2008,9, pp.14-36.

Vester, F., 2007. The Art of Interconnected Thinking: Tools and Concepts for a New Approach to Tackling Complexity. Munich, Germany: MCB (Management, Cybernetics, Bionics) Verlag.

Wasson, B., and Mørch, A.I., 2000. Identifying collaboration patterns in collaborative telelearning scenarios. Educational Technology \& Society 3(3), pp.237-248.

Wenger, E., 1998. Communities of Practice: Learning, Meaning and Identity. Cambridge, UK: Cambridge University Press. 